\title{
Significant concentrations of nitryl chloride sustained in the morning: Investigations of the causes and impacts on ozone production in a polluted region of northern China
}

Yee Jun Tham ${ }^{1}$, Zhe Wang ${ }^{1}$, Qinyi Li $^{1}$, Hui Yun ${ }^{1}$, Weihao Wang ${ }^{1}$, Xinfeng Wang ${ }^{2}$, Likun Xue ${ }^{2}$, Keding $\mathrm{Lu}^{3}$, Nan $\mathrm{Ma}^{4}$, Birger Bohn ${ }^{5}$, Xin Li ${ }^{5}$, Simonas Kecorius ${ }^{4}$ and Johannes Größ ${ }^{4}$, Min Shao ${ }^{3}$, Alfred Wiedensohler ${ }^{4}$, Yuanhang Zhang ${ }^{3}$, and Tao Wang ${ }^{1 *}$.

$10 \quad{ }^{1}$ Department of Civil and Environmental Engineering, The Hong Kong Polytechnic University, Hong Kong, China ${ }^{2}$ Environment Research Institute, Shandong University, Jinan, Shandong, China

${ }^{3}$ State Key Joint Laboratory of Environmental Simulation and Pollution Control, College of Environmental Sciences and Engineering, Peking University, Beijing, China

${ }^{4}$ Leibniz Institute for Tropospheric Research, Permoserstr. 15, 04318 Leipzig, Germany

${ }^{5}$ Forschungszentrum Jülich, Institut IEK-8: Troposphäre, 52425 Jülich, Germany

*Correspondence to: T. Wang (cetwang@ polyu.edu.hk)

Table S1. Validation of meteorological simulations by WRF.

\begin{tabular}{lcccc}
\hline & $\begin{array}{c}\mathrm{T} \\
\left({ }^{\circ} \mathrm{C}\right)\end{array}$ & $\begin{array}{c}\mathrm{RH} \\
(\%)\end{array}$ & $\begin{array}{c}\text { WD } \\
\left({ }^{\circ}\right)\end{array}$ & $\begin{array}{c}\mathrm{WS} \\
(\mathrm{m} / \mathrm{s})\end{array}$ \\
\hline Observation Average & 24.00 & 74.31 & 161.03 & 1.75 \\
Simulation Average & 23.03 & 78.03 & 162.76 & 2.15 \\
mean bias & -0.96 & 3.72 & 1.72 & 0.39 \\
root mean square error & 2.70 & 10.67 & 122.26 & 1.54 \\
\hline
\end{tabular}


Table S2. Mean concentrations and standard deviations ( $\sigma$ ) of each species and meteorological conditions used as input in the box-model simulations. Concentrations are in ppbv unless stated.

\begin{tabular}{|c|c|c|c|c|}
\hline \multirow{2}{*}{ Species } & \multicolumn{2}{|c|}{ Average } & \multicolumn{2}{|c|}{ Megacity case } \\
\hline & Mean & $\pm \sigma$ & Mean & $\pm \sigma$ \\
\hline $\mathrm{ClNO}_{2}$ & 0.19 & 0.16 & 0.52 & 0.65 \\
\hline NO & 1.32 & 1.30 & 0.65 & 0.76 \\
\hline $\mathrm{NO}_{2}$ & 13.11 & 5.96 & 10.37 & 5.28 \\
\hline $\mathrm{O}_{3}$ & 54.3 & 30.2 & 54.6 & 29.1 \\
\hline $\mathrm{SO}_{2}$ & 6.75 & 3.51 & 4.373 & 4.18 \\
\hline $\mathrm{CO}$ & 549 & 63 & 541 & 207 \\
\hline HONO & 0.75 & 0.37 & 0.51 & 0.29 \\
\hline $\mathrm{HCl}$ & 1.15 & 0.40 & 1.26 & 0.93 \\
\hline methane & 2361 & 362 & 2090 & 233 \\
\hline methanol & 24.707 & 5.673 & 15.698 & 4.805 \\
\hline acetaldehyde & 1.887 & 0.357 & 1.349 & 0.383 \\
\hline acetic acid & 5.013 & 0.897 & 2.437 & 0.610 \\
\hline acetone & 4.796 & 0.583 & 3.345 & 0.353 \\
\hline formaldehyde & 7.247 & 1.394 & 3.475 & 0.545 \\
\hline ethane & 2.950 & 0.454 & 2.465 & 0.717 \\
\hline ethene & 1.813 & 0.860 & 1.034 & 0.721 \\
\hline propane & 1.171 & 0.304 & 1.186 & 0.456 \\
\hline propene & 0.310 & 0.170 & 0.221 & 0.161 \\
\hline i-butane & 0.413 & 0.130 & 0.359 & 0.159 \\
\hline n-butane & 0.701 & 0.249 & 0.591 & 0.292 \\
\hline ethyne & 1.490 & 0.282 & 0.835 & 0.267 \\
\hline 1-butene & 0.031 & 0.017 & 0.038 & 0.036 \\
\hline 2-methylpropene & 0.063 & 0.034 & 0.032 & 0.035 \\
\hline trans-2-butene & 0.177 & 0.027 & 0.062 & 0.023 \\
\hline cis-2-butene & 0.015 & 0.006 & 0.011 & 0.011 \\
\hline i-pentane & 0.411 & 0.141 & 0.310 & 0.181 \\
\hline n-pentane & 0.322 & 0.124 & 0.204 & 0.129 \\
\hline 1,3-butadiene & 0.022 & 0.016 & 0.014 & 0.012 \\
\hline 1-pentene & 0.017 & 0.008 & 0.016 & 0.009 \\
\hline isoprene & 0.664 & 0.473 & 0.249 & 0.237 \\
\hline 2,3-dimethylbutane & 0.090 & 0.029 & 0.062 & 0.022 \\
\hline 2-methylpentane & 0.154 & 0.055 & 0.144 & 0.086 \\
\hline
\end{tabular}




\begin{tabular}{|c|c|c|c|c|}
\hline MTBE & 0.121 & 0.045 & 0.073 & 0.048 \\
\hline 3-methylpentane & 0.119 & 0.047 & 0.086 & 0.054 \\
\hline 1-hexene & 0.056 & 0.021 & 0.044 & 0.017 \\
\hline n-hexane & 0.148 & 0.064 & 0.120 & 0.085 \\
\hline methylethylketone & 2.004 & 0.106 & 3.401 & 0.562 \\
\hline 2-methylhexane & 0.033 & 0.012 & 0.028 & 0.017 \\
\hline cyclohexane & 0.051 & 0.022 & 0.042 & 0.027 \\
\hline 3-methylhexane & 0.056 & 0.019 & 0.044 & 0.022 \\
\hline benzene & 1.007 & 0.265 & 0.592 & 0.229 \\
\hline n-heptane & 0.080 & 0.025 & 0.061 & 0.037 \\
\hline toluene & 0.786 & 0.273 & 0.566 & 0.291 \\
\hline n-octane & 0.054 & 0.014 & 0.029 & 0.020 \\
\hline ethylbenzene & 0.198 & 0.066 & 0.160 & 0.089 \\
\hline n-nonane & 0.028 & 0.010 & 0.019 & 0.012 \\
\hline o-xylene & 0.097 & 0.036 & 0.069 & 0.034 \\
\hline styrene & 0.047 & 0.030 & 0.033 & 0.026 \\
\hline m-ethyltoluene & 0.021 & 0.008 & 0.019 & 0.010 \\
\hline 1,3,5-trimethylbenzene & 0.019 & 0.006 & 0.019 & 0.008 \\
\hline 1,2,4-trimethylbenzene & 0.095 & 0.027 & 0.058 & 0.025 \\
\hline 1,2,3-trimethylbenzene & 0.017 & 0.005 & 0.016 & 0.006 \\
\hline Aerosol surface area $\left(\mu \mathrm{m}^{2} \mathrm{~m}^{-3}\right)$ & $1.60 \times 10^{-3}$ & $3.72 \times 10^{-4}$ & $1.24 \times 10^{-3}$ & $4.92 \times 10^{-4}$ \\
\hline Temperature $(\mathrm{K})$ & 300.00 & 3.12 & 296.49 & 3.56 \\
\hline Relative Humidity (\%) & 64 & 12 & 67 & 17 \\
\hline$j_{\mathrm{NO} 2}\left(\mathrm{~s}^{-1}\right)^{a}$ & $3.16 \times 10^{-3}$ & $2.01 \times 10^{-3}$ & $3.33 \times 10^{-3}$ & $2.52 \times 10^{-3}$ \\
\hline$j_{\mathrm{O} 3}\left(\mathrm{O}^{1} \mathrm{D}\right)\left(\mathrm{s}^{-1}\right)^{a}$ & $7.25 \times 10^{-6}$ & $6.36 \times 10^{-6}$ & $7.33 \times 10^{-6}$ & $6.99 \times 10^{-6}$ \\
\hline$j_{\mathrm{HONO}}\left(\mathrm{s}^{-1}\right)^{a}$ & $5.38 \times 10^{-4}$ & $3.45 \times 10^{-4}$ & $5.68 \times 10^{-4}$ & $4.29 \times 10^{-4}$ \\
\hline$j_{\mathrm{CINO} 2}\left(\mathrm{~s}^{-1}\right)^{a}$ & $1.27 \times 10^{-4}$ & $8.06 \times 10^{-5}$ & $1.53 \times 10^{-4}$ & $1.26 \times 10^{-4}$ \\
\hline
\end{tabular}




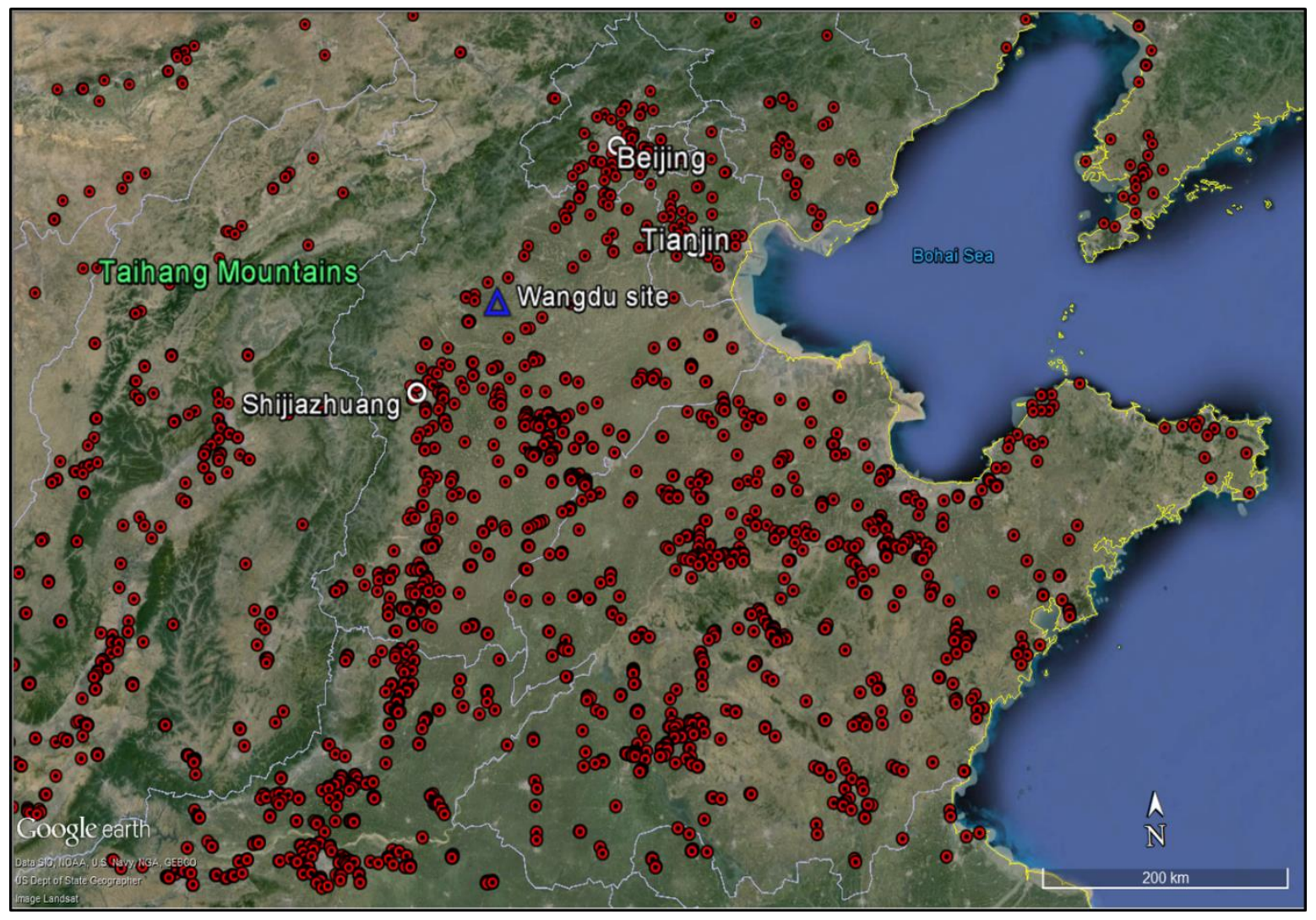

Figure S1. Active fire hotspots (red dots) in NCP from FIRMS covering the study period of 20 June - 9 July 2014 (Data available at https://earthdata.nasa.gov/firms). 
Corona
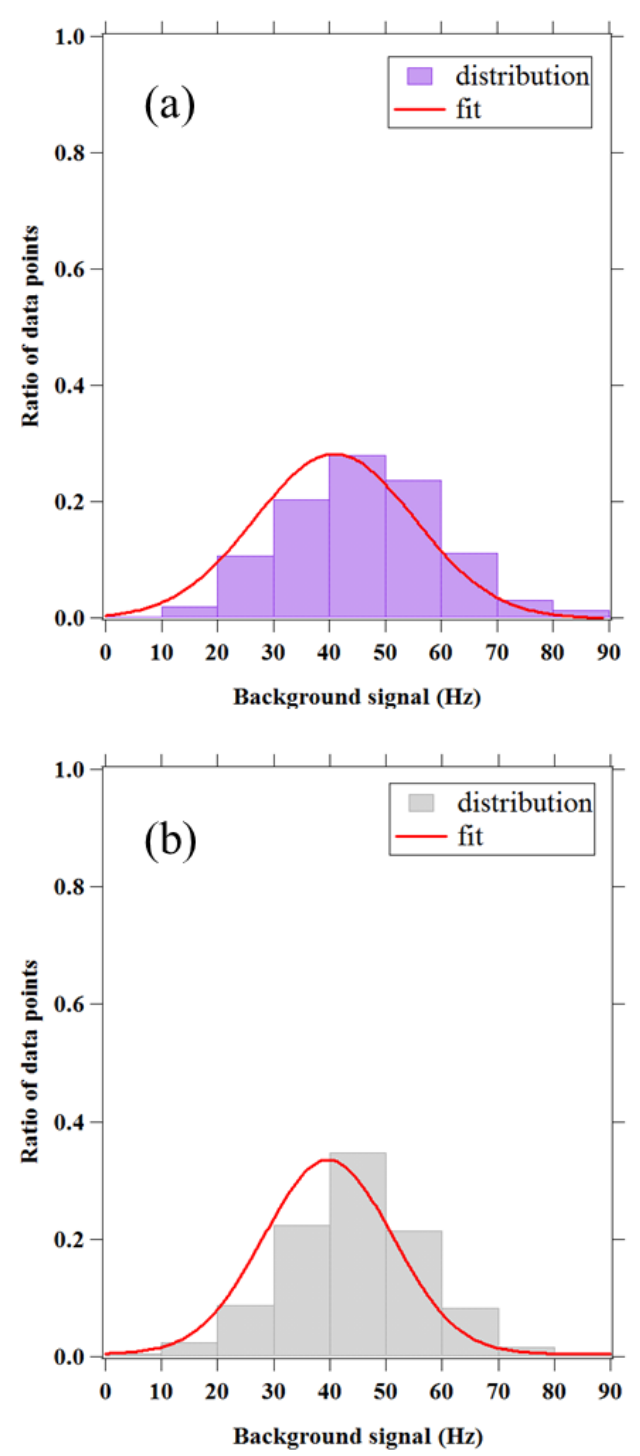

Radioactive
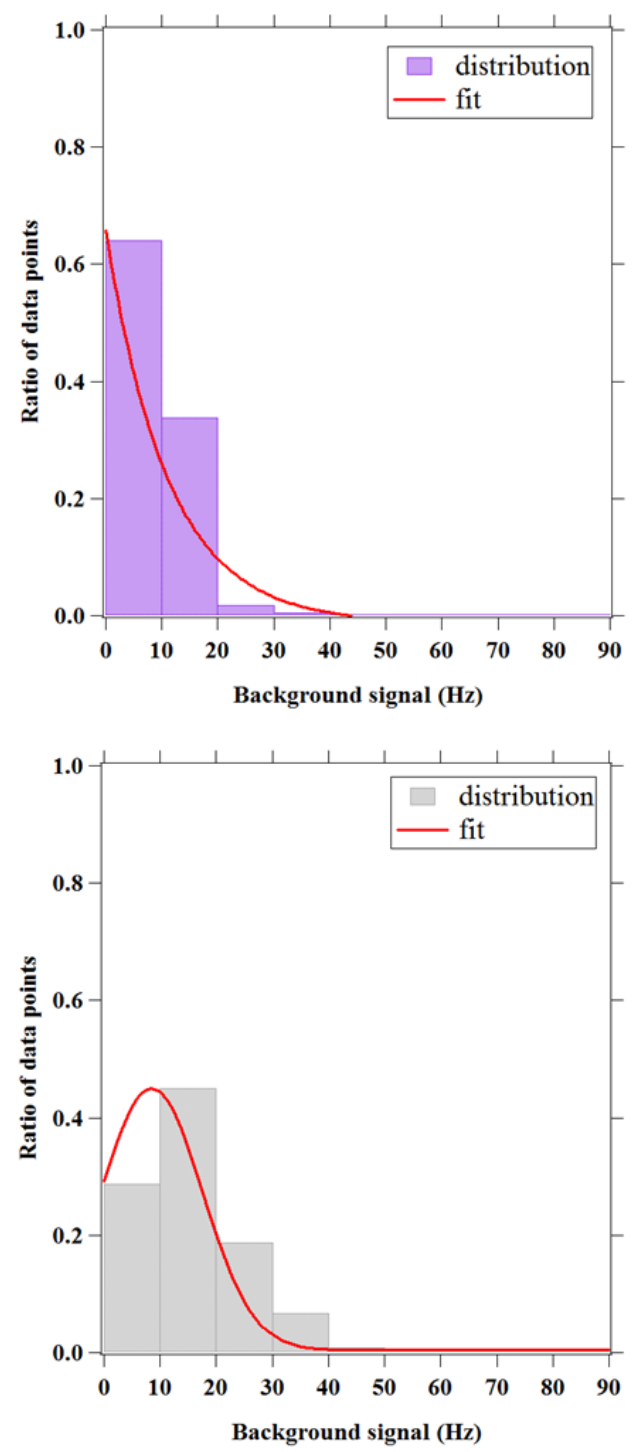

Figure S2. Distributions of ambient background signals of $\mathrm{ClNO}_{2}$ (in purple) and $\mathrm{N}_{2} \mathrm{O}_{5}$ (in grey) from CIMS operated with corona discharge source and ${ }^{210} \mathrm{Po}$ radioactive source $(N=902)$. 

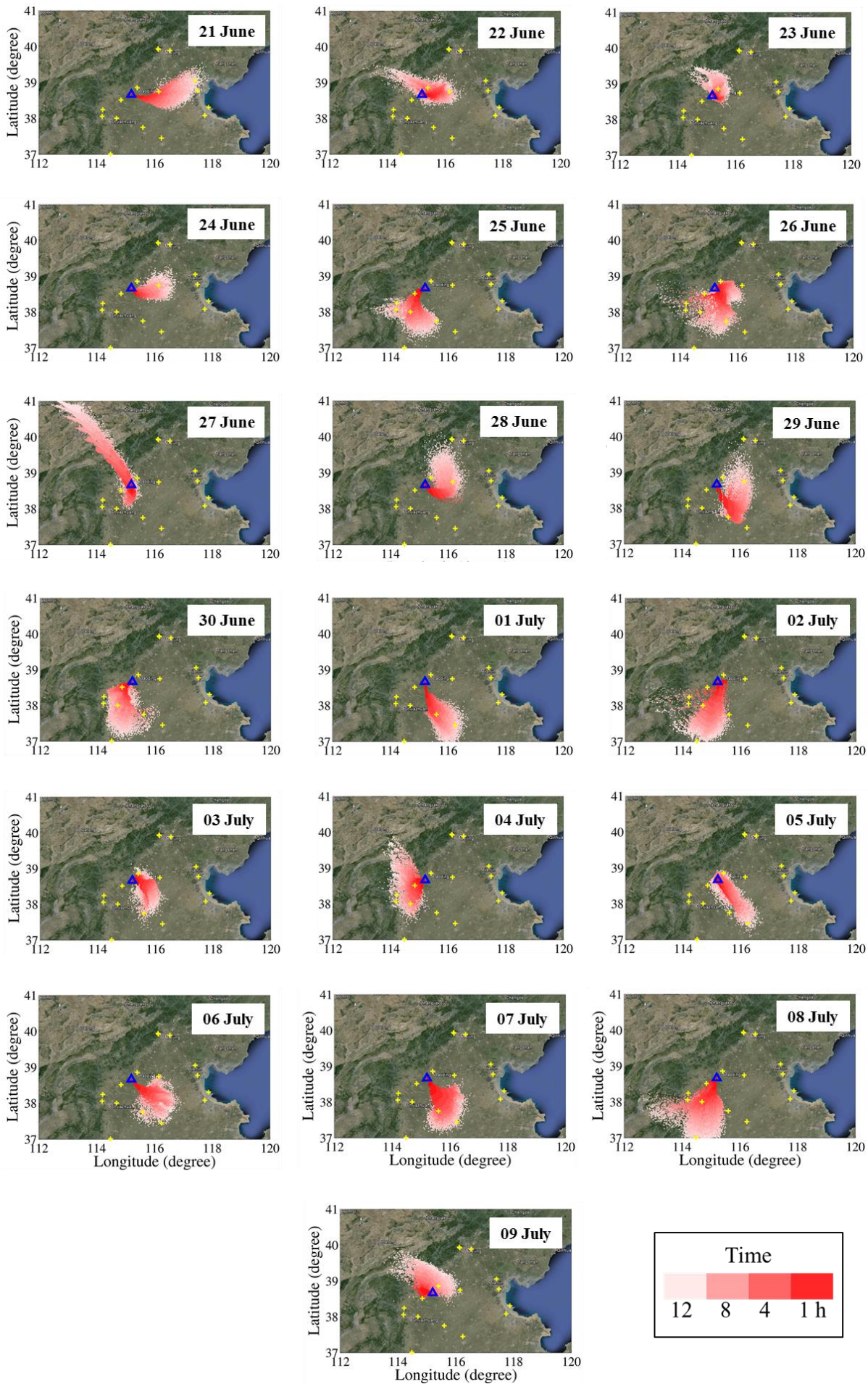
Figure S3. History of air masses that arrived Wangdu sampling site at 00:00.
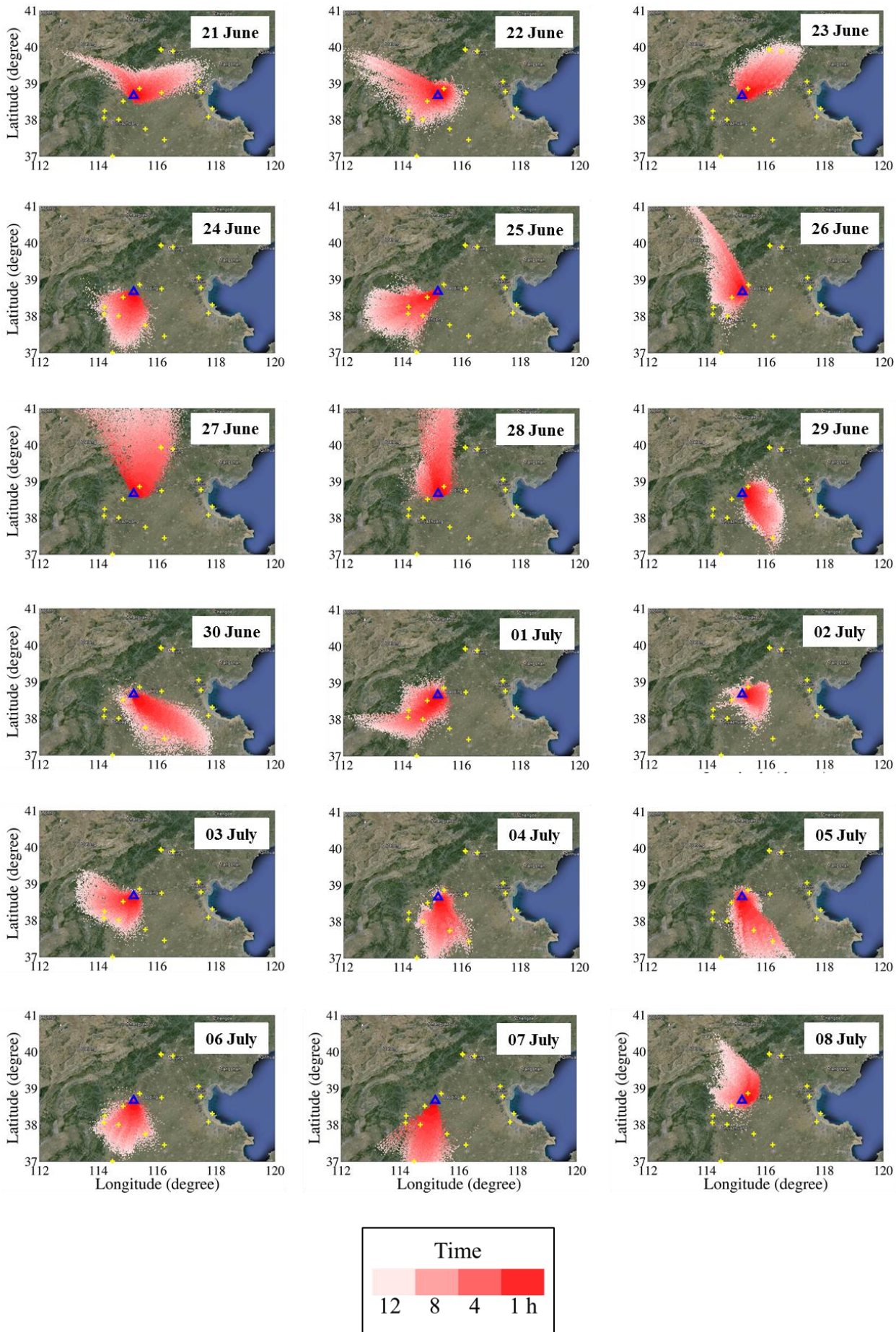
Figure S4. History of air masses that arrived Wangdu sampling site at 14:00.

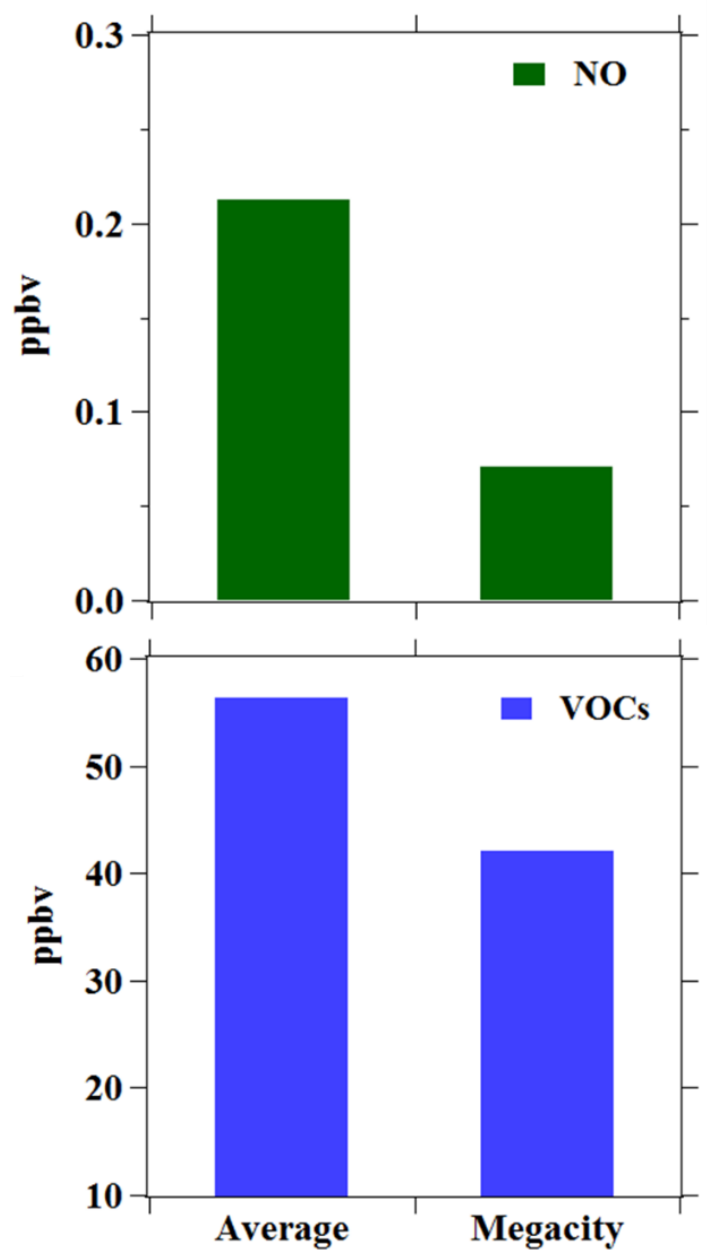

Figure S5. Different level of NO (upper) and total VOCs (lower) between campaign average and the megacity case (averaged between 20:30 to 23:30 of the night). 


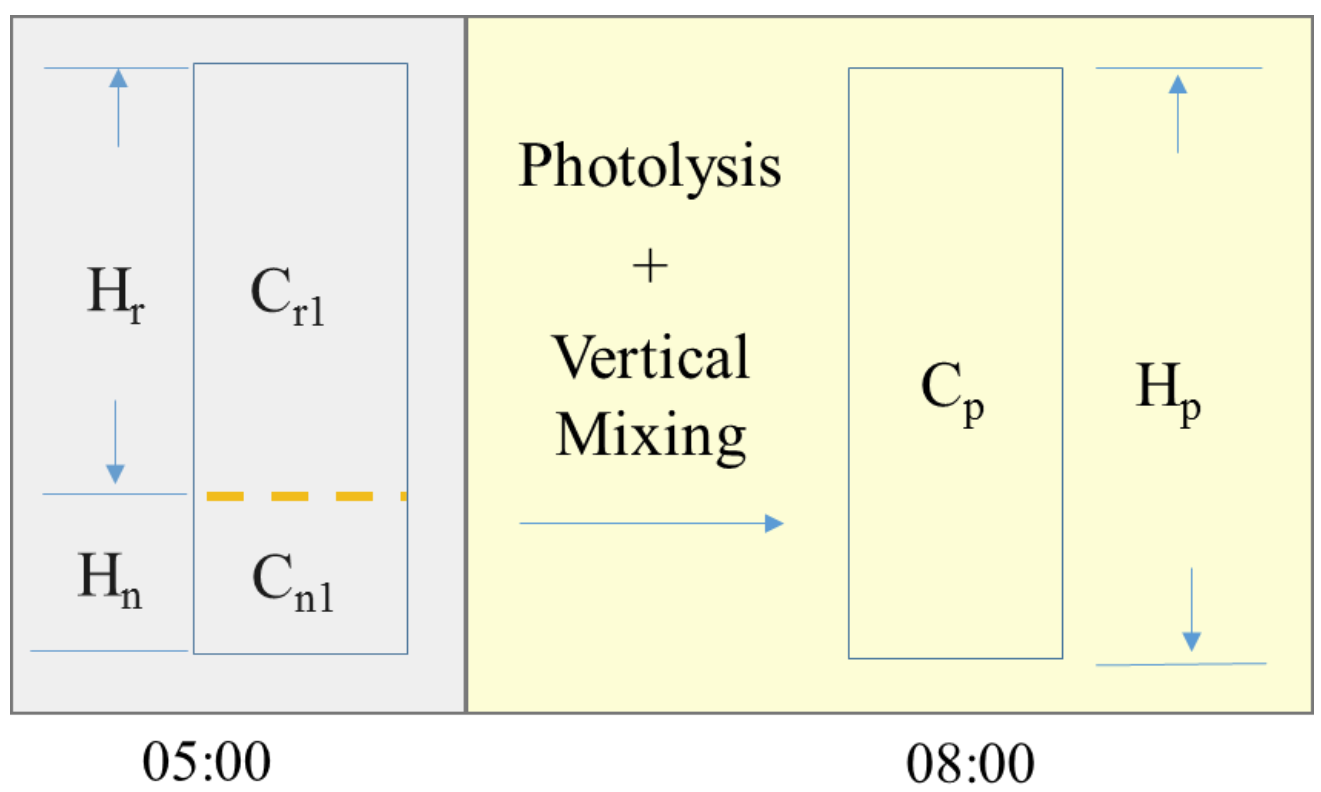

Figure S6. Conceptual diagram of 1-D model used for the estimation of $\mathrm{ClNO}_{2}$ mixing ratios in the RL before sunrise.

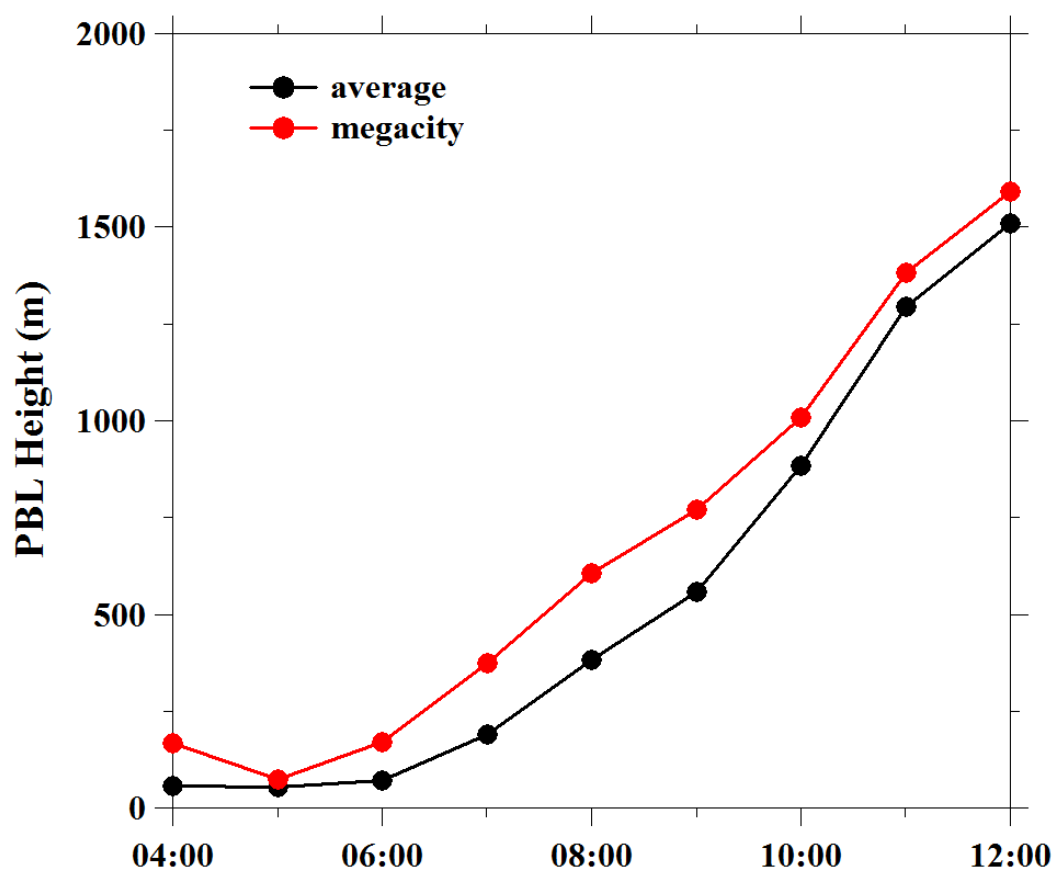

$5 \quad$ Figure S7. WRF simulated PBL heights (a.g.l.) for the campaign average and the megacity case. 

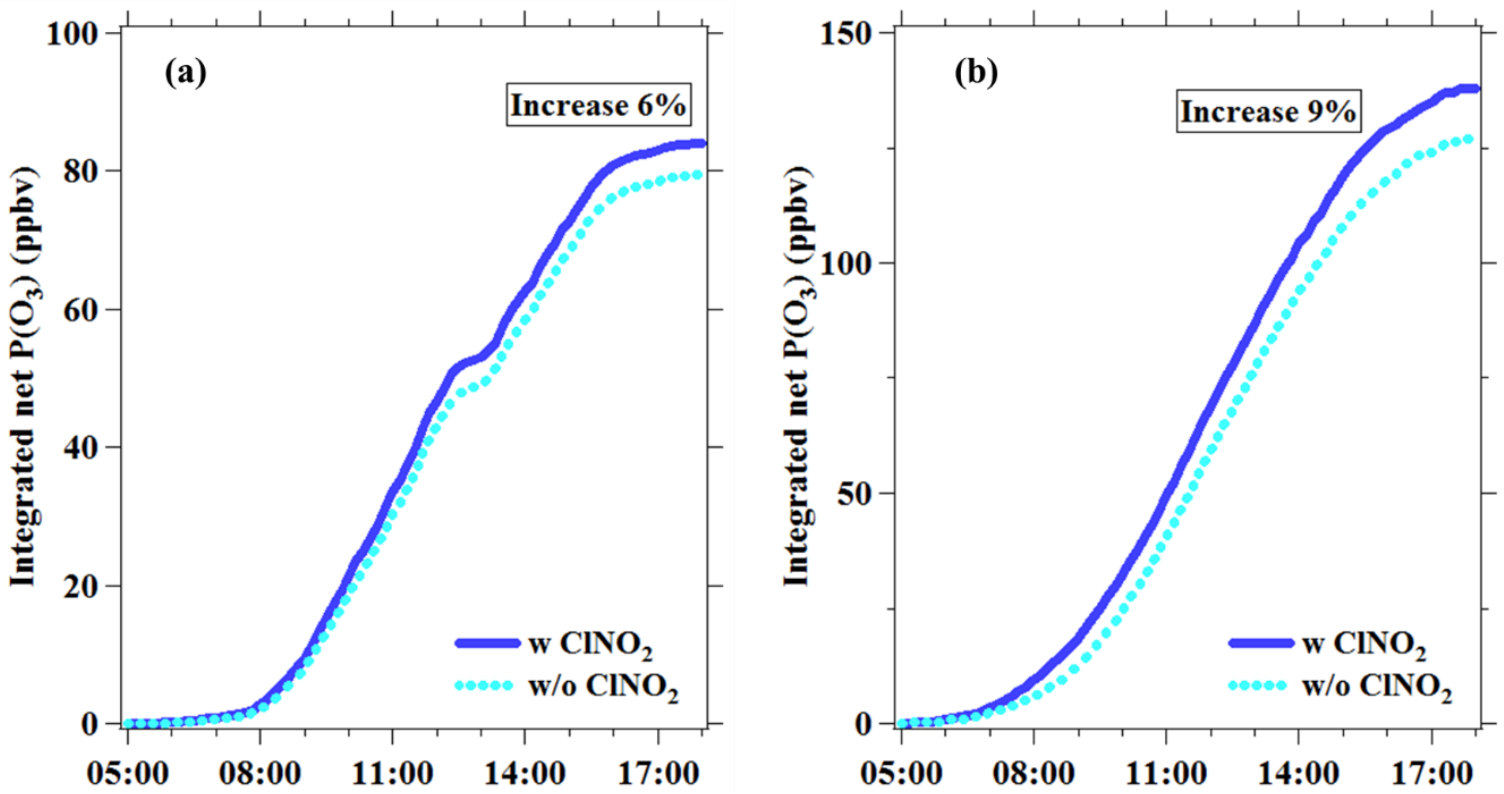

5 Figure S8. Daily integrated net $\mathrm{O}_{3}$ production simulated with and without $\mathrm{CINO}_{2}$ input; (a) the $\mathrm{ClNO}_{2}$ concentration in megacity case was decreased similar to that of average condition, and b) the $\mathrm{ClNO}_{2}$ concentration in average condition was increased to that of megacity case. 


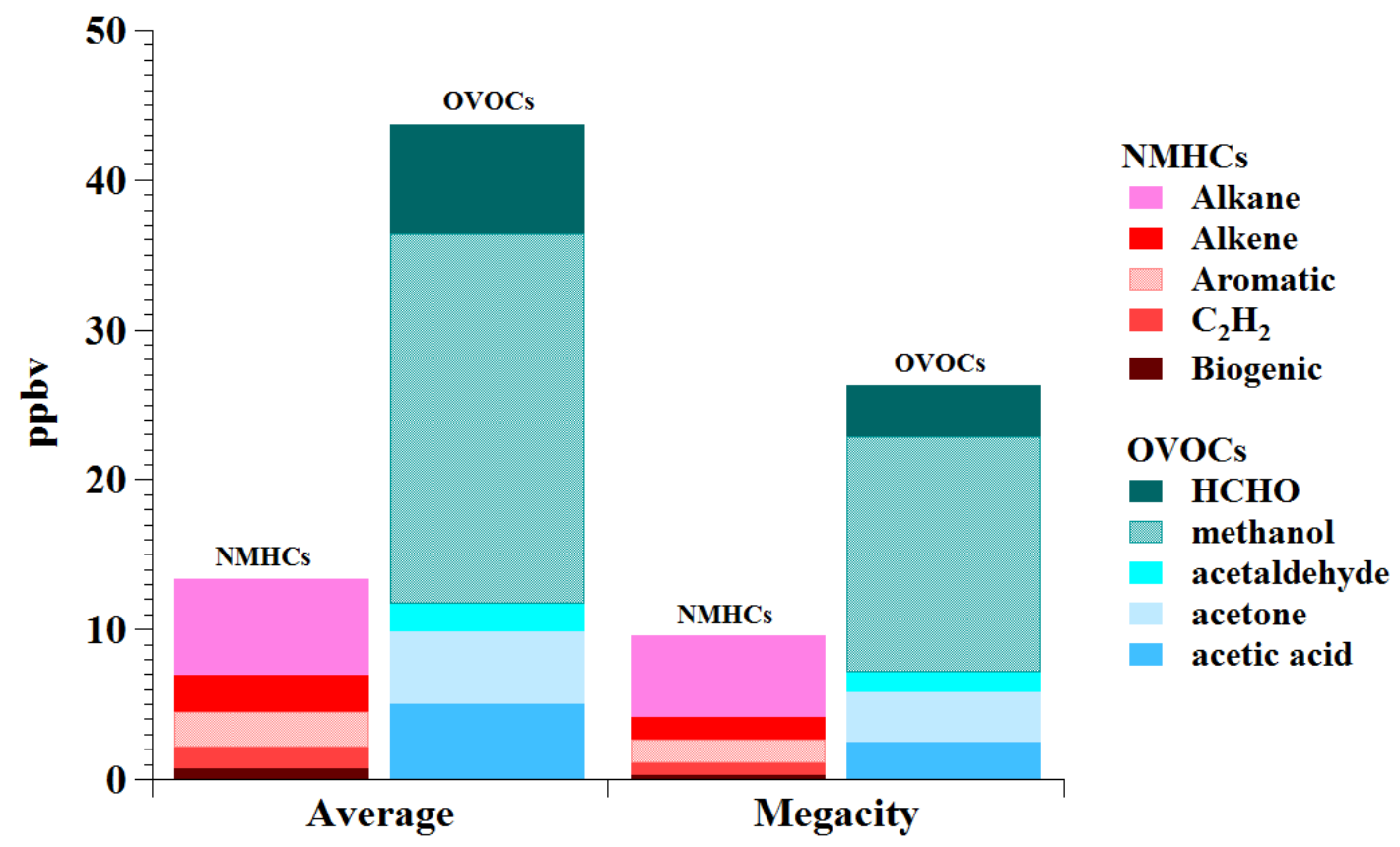

Figure S9. 24-hour average concentrations of different NMHCs and OVOCs groups/species for campaign average and the megacity case.

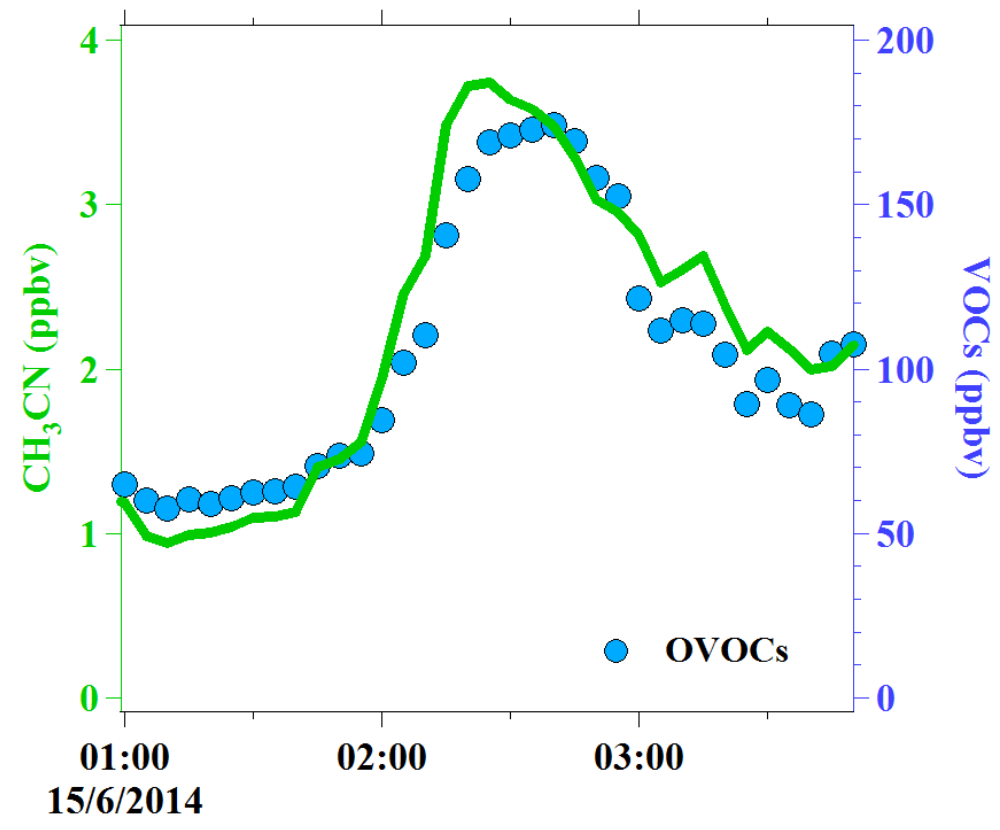


Figure S10. Huge amount of OVOC observed in a fresh biomass burning plume in early morning of 15 June 2014 (as indicated by high $\mathrm{CH}_{3} \mathrm{CN}$ ).

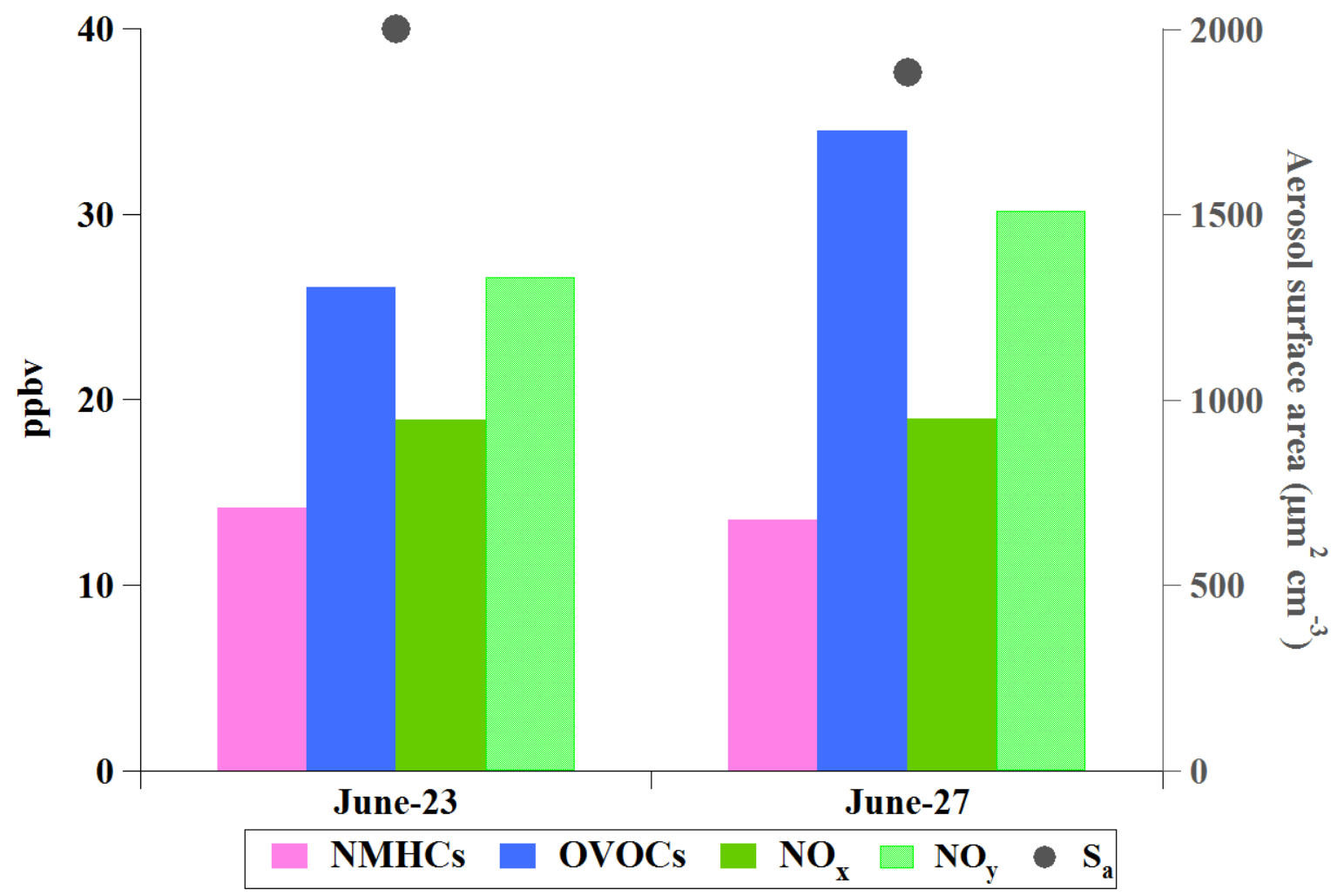

5 Figure S11. Examples of smaller OVOC to NMHC ratios and similar mean mixing ratio of $\mathrm{NO}_{\mathrm{x}}$, $\mathrm{NO}_{\mathrm{y}}$, and $\mathrm{S}_{\mathrm{a}}$ in other cases of megacity outflow from Beijing/Tianjin on 23 and 27 June 2014. 